\title{
Molecular Magnetic Resonance and Ultrasound Imaging of Tumor Angiogenesis
}

\author{
Marie Poirier-Quinot ${ }^{1,2^{*}}$, Ingrid Leguerney ${ }^{1,2}$, Ludovic De Rochefort ${ }^{1,2}$, Stéphanie Pitre-Champagnat ${ }^{1,3}$, \\ Xavier Violas ${ }^{4}$, Sandra Robin ${ }^{1,3}$, Rose-Marie Dubuisson ${ }^{1,2}$, Jean-Christophe Ginefri ${ }^{1,2}$, Philippe Robert ${ }^{4}$, \\ Luc Darrasse $^{1,2}$ and Nathalie Lassau ${ }^{1,3}$
}

${ }^{1}$ Imagerie par Résonance Magnétique Médicale et Multi-modalités - UMR8081(IR4M), CNRS, University Paris-Sud, France

${ }^{2}$ Université Paris-Saclay, France

${ }^{3}$ Institut Gustave Roussy, France

${ }^{4}$ Experimental Imaging, MRI Unit, Research Division, Guerbet, France

*Corresponding author: Marie Poirier-Quinot, Imagerie par Résonance Magnétique Médicale et Multi-modalités UMR8081(IR4M), CNRS, University Paris-Sud; Université Paris-Sud, Bâtiment 220, 91405 ORSAY, France, Tel: +33-1-6915-79-65, Fax: +33-1-69-15-41-36, E-mail: marie.poirier-quinot@u-psud.fr

\begin{abstract}
Seeking out and identifying imaging biomarkers for early cancer diagnosis and the evaluation of patient response to therapy requires an improvement in the specificity of imaging techniques. This study explores in vivo neo-angiogenesis assessment using molecular mechanisms through target molecular Magnetic Resonance (MR) and Ultrasound (US). In this context, our study examines and compares the use of both imaging technics, targeting the same integrin in a mouse xeno graft tumor model. Following xeno transplantation of human renal cell carcinoma (Human A498), thirteen nude mice were injected with avß3-targeted and non-targeted Contrast Agents (CA) for MR and US use, respectively. CA binding to the targeted receptor was measured through Dynamic Susceptibility Contrast MR imaging and Differential Targeted Enhancement (DTE) US imaging. The specificities and co location of both targeted CAs were studied throughout the tumor, in both hypoand hyper-vascularized areas.

One hour postinjection, a significant difference was observed between the signals of targeted and non-targeted MR CA $(p=0.03)$. The DTE-US targeted CA was significantly enhanced compared to non-targeted CA ( $p=0.002)$. In the hypo- and hyper-vascularized regions of interest, a strong correlation was observable between both modalities, with values of $r_{10 \min }=0.86(p=0.0003), r_{30 \min }=0.85(p=$ $0.0004)$ and $r_{60 \text { min }}=0.87(p=0.0001)$.

This study highlights the specificity of each targeted CA. A high correlation was noted between MR and US molecular imaging for angiogenesis assessment. These two molecular imaging modalities may be used interchangeably to monitor patient response to anti-angiogenic treatment.
\end{abstract}

\section{Keywords}

Bimodal molecular imaging DCE-US/MRI, $\mathrm{Av}_{3}-$ Targeted contrast agent, USPIO nanoemulsion, US microbubbles

\section{Abbreviations}

CA: Contrast Agents; US: Ultrasound; DTE: Differential Targeted Enhancement; MR: Magnetic Resonance; SE: Standard Error; Fps: Frames per second; DSC: Dynamic Susceptibility Contrast; ROI: Region of Interest

\section{Introduction}

Molecular imaging using targeted contrast agents has been used to highlight fundamental biological mechanism for some time now, and facilitates the development of new drugs [1]. It has also proved to be a valuable tool for the early detection of molecular and cellular events, and for the characterization of molecule expression levels involved in inflammation and angiogenesis process [2-5]. The development of new contrast agents in the oncology field focuses on their specific targeting of angiogenesis and their binding to receptors/ molecules such as the $\alpha v \beta 3$ integrin, which is highly expressed on activated endothelial cells in tumor vessels [6].

Molecular Ultrasound (US) is an recent imaging approach that has rapidly found its niche among other molecular imaging modalities thanks to its high tempo-

Citation: Poirier-Quinot M, Leguerney I, De Rochefort L, Pitre-Champagnat S, Violas X, et al. (2017) Molecular Magnetic Resonance and Ultrasound Imaging of Tumor Angiogenesis. Int J Radiol Imaging Technol 3:027. doi.org/10.23937/2572-3235.1510027

Received: June 26, 2017: Accepted: October 09, 2017: Published: October 11, 2017

Copyright: (c) 2017 Poirier-Quinot M, et al. This is an open-access article distributed under the terms of the Creative Commons Attribution License, which permits unrestricted use, distribution, and reproduction in any medium, provided the original author and source are credited. 
Table 1: Summary of experimental protocol - evaluation of MR and US CA specificity - evaluation of targeted MR and US CA colocalization.

\begin{tabular}{|c|c|c|c|c|}
\hline \multirow[t]{2}{*}{ Mouse } & \multicolumn{2}{|l|}{ MR } & \multicolumn{2}{|l|}{ US } \\
\hline & Non-targeted CA & Targeted CA & Non-targeted CA & Targeted CA \\
\hline 1 & $x$ & + & & \\
\hline $2\left(^{*}\right)$ & $x$ & $x$ & $x$ & $x$ \\
\hline 3 & + & $x$ & & $x$ \\
\hline $4\left(^{*}\right)$ & $x$ & $x$ & $x$ & $x$ \\
\hline 5 & + & $x$ & & $x$ \\
\hline $6\left(^{*}\right)$ & $x$ & $x$ & $x$ & $x$ \\
\hline 7 & $x$ & + & & \\
\hline 8 & & & $x$ & \\
\hline 9 & & & $x$ & \\
\hline 10 & & & $x$ & \\
\hline
\end{tabular}

"Same mice for US specificity, MR specificity, and targeting protocols; × Same mice for MR and US CA co-localization; + Dead mouse.

ral resolution and ease of use [2]. Dynamic Susceptibility Contrast (DSC)-Magnetic Resonance (MR) imaging uses Ultra-Small Super Paramagnetic Iron Oxide (USPIO) Contrast Agent (CA) to provide information on tumor vascularization and hemodynamic status.

With the development of targeted agents binding to specific receptors, DSC-MR imaging can also provide molecular information in intra- and extra-vascular conditions; this has the advantage of requiring low CA concentrations making it possible to probe specific molecular events [7].

The increasing development of novel targeted therapies to treat tumors necessitates a parallel development of technologies that can determine their potential efficacy, and thus facilitate therapeutic protocol planning. Efforts are also being made to assess and quantify biomarkers of treatment response that could provide information on the location and extent of cancer, including tissue characteristics or spatial heterogeneity $[2,8]$.

The aim of this work was to evaluate the feasibility of detection of molecular events and compare in vivo quantitative US and MR molecular imaging protocols.

The pilot study was performed on renal cell carcinoma xeno grafts in mice and was designed with two main goals in mind: 1) To evaluate the specificity of two contrast agents (MR iron oxide nanoemulsion and US microbubbles), both of which were specifically designed to target $\alpha v \beta 3$ integrin and 2) To compare the colocalization of molecular expression of the targeted integrin obtained by specific MR and US contrast agent acquisitions, respectively (summarized in Table 1).

\section{Materials and Methods}

All experiments followed the guidelines of the European Convention for the Protection of Vertebrate Animals used for experimental and other scientific purposes, and were approved by the Animal Research Committees of the relevant laboratories, C2EA-26 and C2EA-44 (both registered at the French research ministry).

\section{Mice and tumor model}

Ten female immune deficient nude mice (6-8 weeks old) were bred and housed in the Animal Care Facility at the Institute Gustave Roussy (Villejuif, France), in accordance with institutional guidelines for animal welfare. Human A498 renal cell carcinoma (ATCC-HTB-44, American Type Culture Collection, Manassas, USA) were cultivated in EMEM (Eagle's Minimum Essential Medium, Gibco Life Technologies, Gaithersburg, MD, USA) supplemented with 10\% FBS (Fetal Bovine Serum). The experiments started 30 days after $3 \times 10^{6}$ cells in $0.2 \mathrm{~mL}$ matrigel (Corning Corporated, Tewksbury, USA) were subcutaneously inoculated into the right flank of the mice.

\section{Imaging protocol}

Imaging sessions were performed using MR and US, each combined with targeted and non-targeted CA that had been injected intravenously in the systemic circulation (Figure 1). Targeted and non-targeted protocols were performed on the same day (D1) for US experiments, and systematically at a 2-day interval for MR experiments (i.e. non-targeted contrast agent injection was made on D3 or D5 and finally the targeted contrast agent injection was carried out on D3 or D5) to avoid any interaction between the MR- and US-targeted CAs.

Mice were anesthetized before each imaging session by the inhalation of isoflurane (2\%) in room air (1.5 $\mathrm{L} / \mathrm{min}$ ). Their body temperature was monitored and maintained constant using a heated platform during US acquisitions or a constant warm air flow during MR acquisitions. Table 1 shows the number of mice used to evaluate $\alpha v \beta 3$-targeted contrast agents and characterize their affinity to the $\alpha v \beta 3$ sites.

\section{Molecular MR imaging}

MR imaging was performed using a clinical $1.5 \mathrm{~T}$ scanner (Achieva, Philips Healthcare, Best, The Netherlands) equipped with a $60 \mathrm{~cm}$ bore and a conventional $23 \mathrm{~mm}$ diameter microscopy surface coil for reception. Relaxation rate $R_{2}{ }^{*}$ measurements were made using a 3D $\mathrm{T}_{2}{ }^{*}$-weighted gradient-echo multi-echo sequence, selected for its sensitivity to apparent transverse relaxation effects induced by USPIO. Dynamic Susceptibility 


\section{(a) DTE-US}

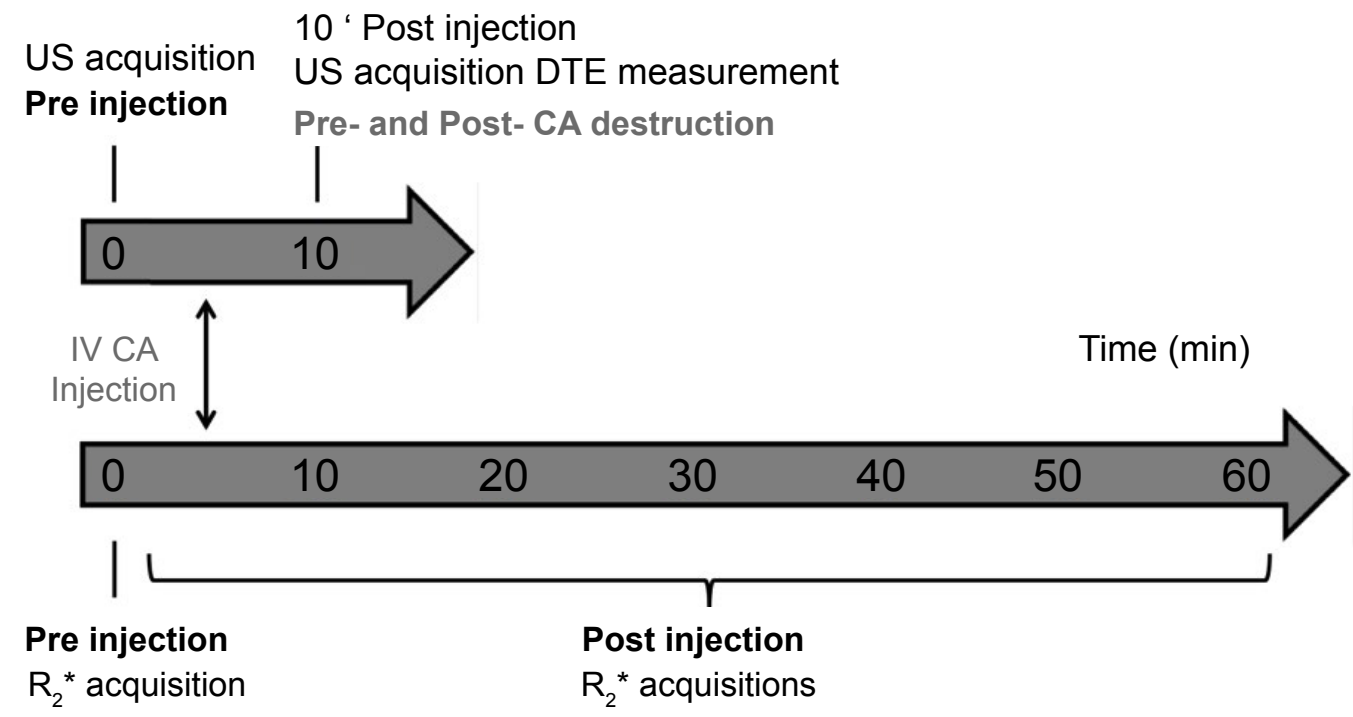

\section{(b) DSE-MRI}

Figure 1: Experimental (a) US; (b) MR protocols. The US protocol began with the CA injection. Ten minutes after the bolus injection, series of images were recorded for approximately 30 seconds. Using the destruction/replenishment approach, a continuous high-powered destructive pulse was then applied to destroy all microbubbles within the US beam elevation. Images were recorded while freely circulating CA replenished the tumor vessels. The MR protocol began with localization and included a repeated $3 D R_{2}{ }^{*}$-weighted sequence for $C A$ quantification. The $R_{2}{ }^{*}$-weighted sequence was then performed pre-contrast $(\mathrm{t}=0 \mathrm{~min})$, and was repeated every 7 minutes until 1 hour post-administration.

Contrast (DSC)-MR was performed repeatedly for one hour after the CA injection. $\mathrm{R}_{2}^{*}$ mapping (3D $0.5 \mathrm{~mm}$ isotropic sequence, $\mathrm{TR} / \mathrm{TE} / \mathrm{ech}$ spacing $=90 / 5.9 / 9.7 \mathrm{~m}$ sec, 6 echoes, $0.3 \times 0.3 \times 0.5 \mathrm{~mm}^{3}$ reconstruction voxel size, $220 \mathrm{~Hz} / \mathrm{pix}$, Tacq $=4.2 \mathrm{~min}$ ) was carried out before and after the injection in the caudal vein of $100 \mu \mathrm{mol}$ $\mathrm{Fe} / \mathrm{kg}$ USPIO-based nanoemulsion functionalized with RGD-binding $\alpha v \beta 3$ (P4000, referred to as E1 in patent application US 2015/0320889 A1, entitled 'Vectorised magnetic emulsion', and filed on Nov. 12, 2015). Identical $R_{2}{ }^{*}$ mapping protocol was also carried out before and after the injection of a non-functionalized control nanoemulsion (P3999, referred to as E1T in patent application US 2015/0320889). Nanoparticle diameters were approximately $190 \mathrm{~nm}$. These contrast agents are also commercialized by Chematech (France). As generally assumed in a first approximation, transverse relaxation rate $R_{2}{ }^{*}=1 / T_{2}{ }^{*}=R_{2,0}{ }^{*}+r_{2}{ }^{*}[\mathrm{Fe}]$ is linear with concentration, and relaxivity is $r_{2}^{*} \sim 210 \mathrm{mM}^{-1} . \mathrm{s}^{-1}$ (patent application US 2015/0320889 A1). All images were processed with Matlab software (2011B, Math works, USA). Mean pre- and post-injection variations, or $\Delta \mathrm{R}_{2}{ }^{*}\left(\mathrm{R}_{2 \text { pre }}{ }^{*} \mathrm{R}_{2 \text { post }}{ }^{*}\right)$, were calculated and monitored for 1 hour to quantify CA concentration, which directly reflects the binding to the targeted receptor.

\section{Contrast-enhanced molecular US}

All US acquisitions were performed on a dedicated system for small animal imaging (VEVO 2100 , Visual Sonics, Canada), using a linear array probe with a 21
$\mathrm{MHz}$ center frequency (MS-250, Visual Sonics). Tumor volumes were measured using 2D B-mode imaging, as described in the literature [9]. 2D Differential Targeted Enhancement Ultrasound (DTE-US) imaging sessions were performed on the maximal transverse section of each tumor using contrast pulse sequencing technology, to reach the nonlinear signal produced by microbubbles. Images were collected at a high spatial resolution (lateral and axial resolution of 165 and $75 \mu \mathrm{m}$, respectively), with $4 \%$ transmission power, 5 frames per second (fps), and a dynamic range of 40-dB [9].

Micro Marker avß3-targeted and control (IgG) microbubbles (Visual Sonics, Toronto) with a diameter of approximately 2-3 $\mu \mathrm{m}$ were manually injected via the retro-orbital vein $(50 \mu \mathrm{L}$ per injection, injection time: 2 seconds). A 30-minute interval between the two injections allowed the first CA to be cleared from the vasculature [9]. After each CA injection, the signal intensity was recorded as a function of time during the wash-in contrast enhancement. CA targeting was then observed 10 minutes after the bolus injection in order to allow the targeted microbubbles to bind to their endothelial molecular receptors [9], and a series of images was recorded over 30 seconds. Using the destruction/replenishment approach [10], a continuous high-powered destructive pulse (100\% transmit power for $1 \mathrm{~s})$ was then applied to destroy all microbubbles within the US beam elevation. Images were recorded while freely circulating CA replenished the tumor vessels. Data were then ana- 


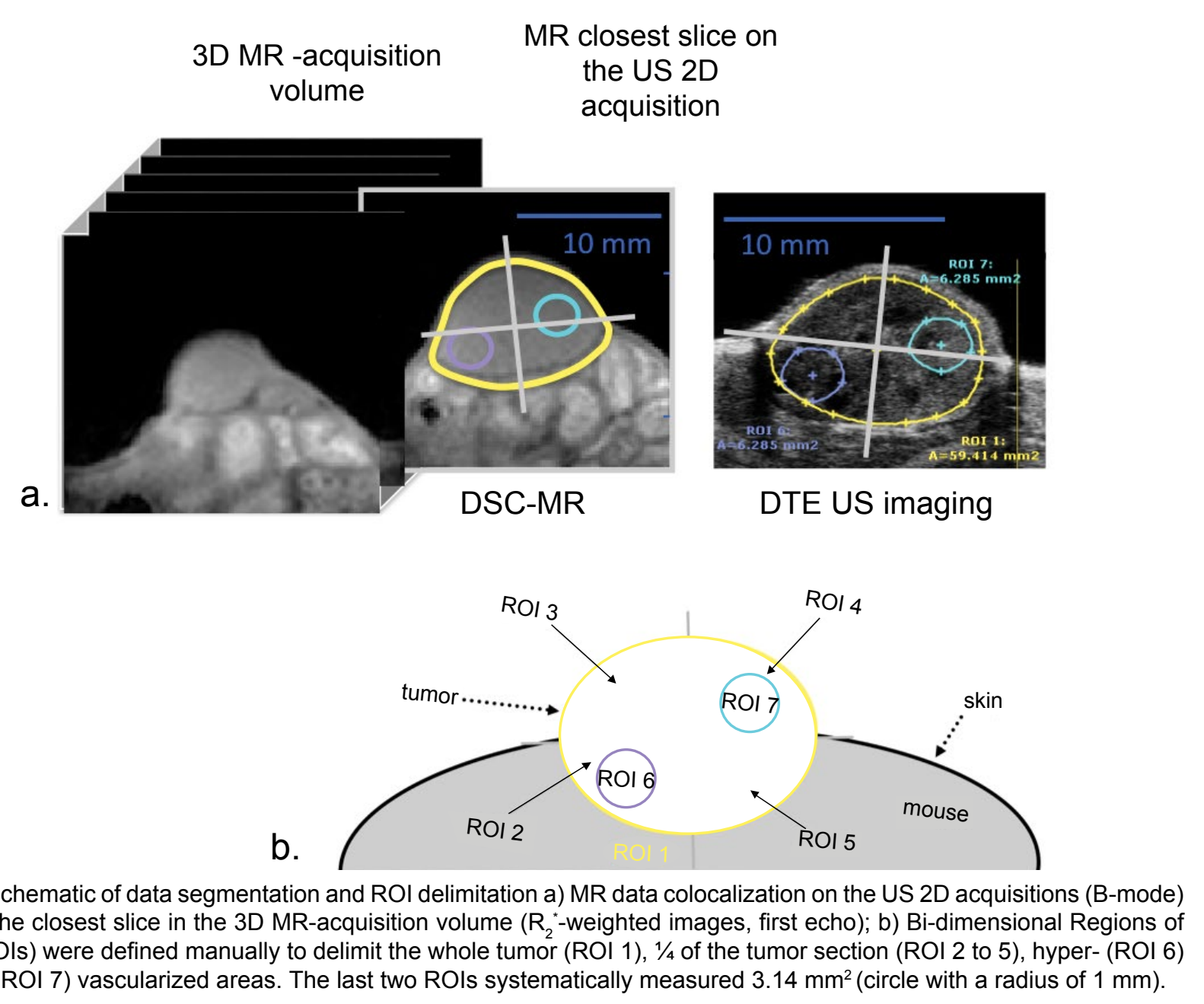

lyzed using the VEVO 2100 software to quantify the DTE value (arbitrary unit) within specific Regions of Interest (ROIs), calculated by using the difference between the mean pre- and post-destruction signal intensities. DTE value is an indicator of the number of microbubbles that adhere to molecular endothelial receptors.

\section{Evaluation of targeted MR and US contrast agent colocalization}

MR data were colocalized on the US 2D acquisitions to choose the closest slice in the 3D MR-acquisition volume $\left(\mathrm{R}_{2}{ }^{*}\right.$-weighted images), (Figure 2a). To compare DTE-US imaging to DSC-MR imaging, mean values of DTE and relaxation rates $\Delta R_{2}{ }^{*}$ were determined in whole tumor ROI (ROI-1) and multiple intratumoral ROI (ROI 2 to 7) to reflect the heterogeneity of intratumoral molecular expression. ROI 2 to 5 was manually drawn to facilitate the location (Figure $2 \mathrm{~b}$ ). The two remaining ROIs (ROI 6 and 7) were determined as the two extrema of targeted contrast agent imaging, i.e. they were hypo(ROI-6) and hyper- (ROI-7) contrast enhanced. ROls all measured $3.14 \mathrm{~mm}^{2}$ (circle with a radius of $1 \mathrm{~mm}$ ).

\section{Immunofluorescence}

Immediately after the last MR imaging session, all five tumors were extracted, embedded in a OCT gel-like medium consisting of polyethylene glycol and polyvinyl alcohol (Cryomatrix OCT compound, Thermo Fisher Scientific, USA), then frozen in liquid nitrogen. The tumors were cut into 7-8 $\mu \mathrm{m}$-thick sections located as close as possible to the maximal transverse section imaged by DTE-US and DSC-MR. Sections were stained with Hematoxylin, Eosin and Saffron (HES) and observed to verify tissue integrity.

To verify the presence of $\beta 3$ on the cells of the vascular endothelium of renal carcinoma, cover slips were incubated overnight at $4{ }^{\circ} \mathrm{C}$ with anti-human rabbit $\beta 3$ (1:1000; 04-1060, Millipore) in TNB buffer as a primary antibody, then incubated for $2 \mathrm{~h}$ with HRP-conjugated goat anti-mouse IgG (1:1000; 111-036-047, Jackson ImmunoResearch) in TNB buffer as a secondary antibody. Given that the tumor tissue could also express the $\beta 3$ integrin, $\beta 3 /$ CD31 double labeling was carried out to demonstrate the labeling of the integrin in the vascular endothelium. CD31 was revealed using anti-mouse rat CD31 (1:50; 553370, BD Biosciences) then goat anti-rat IgG coupled to Alexa Fluor 555 (1:200; A-21434, Life Technologies). The $\beta 3$ was amplified using the TSA kit (NEL749A001KT, Perkin Elmer) and revealed by FITC-Streptavidin (1:200, STAR2B, AbD Serotec). The nuclei were labeled with DAPI (Atlantic Bone Screen, France). Nuclear staining with DAPI allowed the identification of tissue structure and quality. Tissue fluorescence was observed under a multiphoton microscope 


\section{DTE-US}

Pre-injection

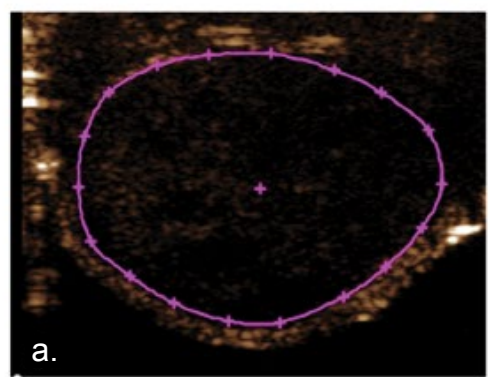

Post-injection

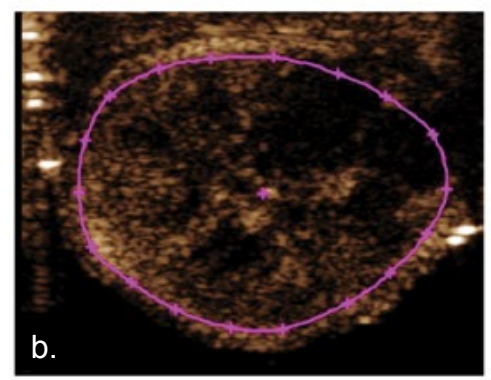

DSC-MR
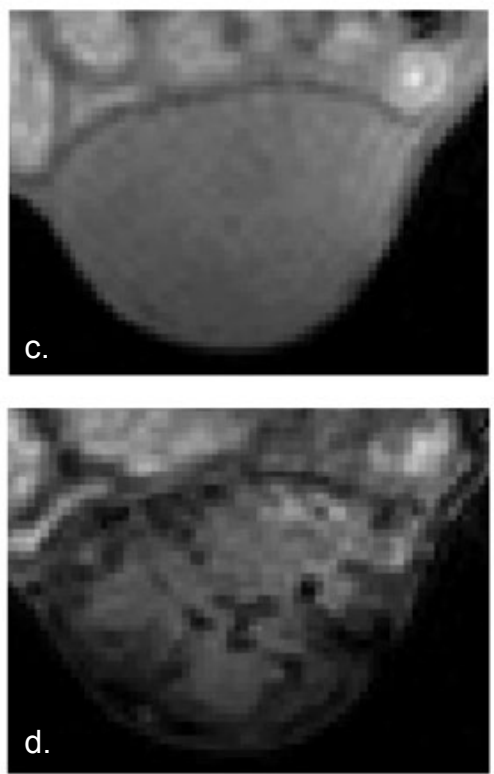

Figure 3: Molecular US imaging in harmonic mode carried out a) pre-injection; b) 10 minutes postinjection of targeted microbubbles. First echo of DSC-MR imaging carried out; c) preinjection; d) 10 minutes postinjection of targeted USPIO nanoemulsion. The MR hyposignal is due to the presence of USPIO nanoemulsion, while microbubbles lead to a hypersignal on US acquisition.

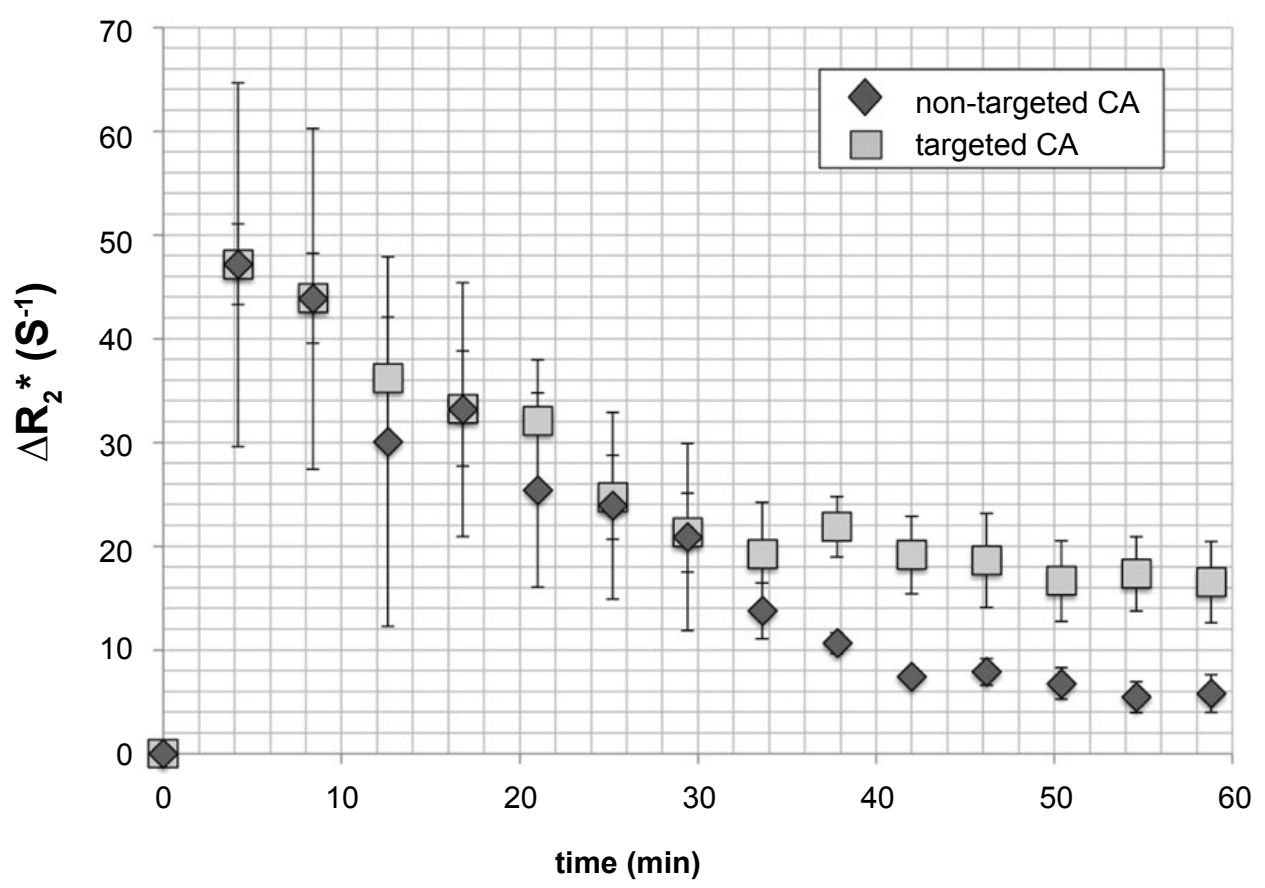

Figure 4: $\Delta R_{2}{ }^{*}$ mean values ( \pm Standard Error SE), for targeted (square) and non-targeted (diamond) CA as a function of time. $\Delta R_{2}{ }^{*}$ values became significantly different between targeted and non-targeted CA for all acquisitions performed every 7 minutes between 37 and 60 minutes postinjection ( $p=0.03$, Wilcox on test for paired samples), thus providing the most appropriate imaging window to image specific binding. At 1 hour postinjection, $\Delta \mathrm{R}_{2}^{*}$ measurement for targeted $C A$ was $16.5 \pm 3.9 \mathrm{~s}^{-1}$, significantly different from the values recorded for non-targeted CA, namely $5.8 \pm 1.8 \mathrm{~s}^{-1}(p=0.03$, Wilcox on test for paired samples).

(SP8, Leica). Regions of interest were photographed at a magnification of $x 63$ through an oil immersion lens.

\section{Statistical analysis}

All continuous measurements were expressed as mean \pm standard deviation. Correlations were statisti- cally analyzed using the Pearson's correlation coefficient ( $r$ ) for normally distributed data, and the Spearman rank coefficient correlation was used in the absence of normal distribution. The significance level of the correlation was determined with corresponding Pearson and Spearman tests. The $\Delta \mathrm{R}_{2}^{*}$ and DTE values were not nor- 
mally distributed and were therefore compared using a non-parametric Wilcox on signed-rank test. A p-value of less than 0.05 was considered statistically significant.

\section{Results}

Figure 3 shows an example of the same tumor imaged by $M R\left(R_{2}^{*}\right.$-weighted scan, first echo with a Signal to Noise Ratio of 70) and DTE-US before and after the targeted CA injection. $S$ although the signal was homogeneous before injection in both MR and US images, strong heterogeneity was observed after the injection of the CA, with signal enhancement in US sessions and an $\mathrm{R}_{2}{ }^{*}$ increase in MR imaging indicating molecular $\alpha \vee \beta 3$ expression.

\section{MR and US contrast agent specificity}

DTE-US measurements at the tenth minute postinjection were compared to $\Delta \mathrm{R}_{2}^{*}$ measurements post-in- jection from 10 to 60 minutes at intervals of 7 minutes, in order to determine the optimal time at which MR imaging parameters should be compared in terms of CA binding specificity. Figure 4 shows the $\Delta R_{2}^{*}$ mean values ( \pm Standard Error SE) for targeted (square) and non-targeted (diamond) CA as a function of time. $\Delta R_{2}{ }^{*}$ values became significantly different between targeted and non-targeted CA for all acquisitions performed 37 minutes postinjection and later $(p=0.03)$, thus providing the imaging window to capture specific binding. At 1 hour postinjection, the $\Delta R_{2}{ }^{*}$ value for targeted CA was $16.5 \pm 3.9 \mathrm{~s}^{-1}$; significantly different to that of non-targeted $C A$, i.e. $5.8 \pm 1.8 \mathrm{~s}^{-1}(\mathrm{p}=0.03)$.

Figure 5 shows the mean ( \pm SE) US contrast signal intensities evaluated 10 minutes after the CA injection, before and after targeted and non-targeted CA destruc-

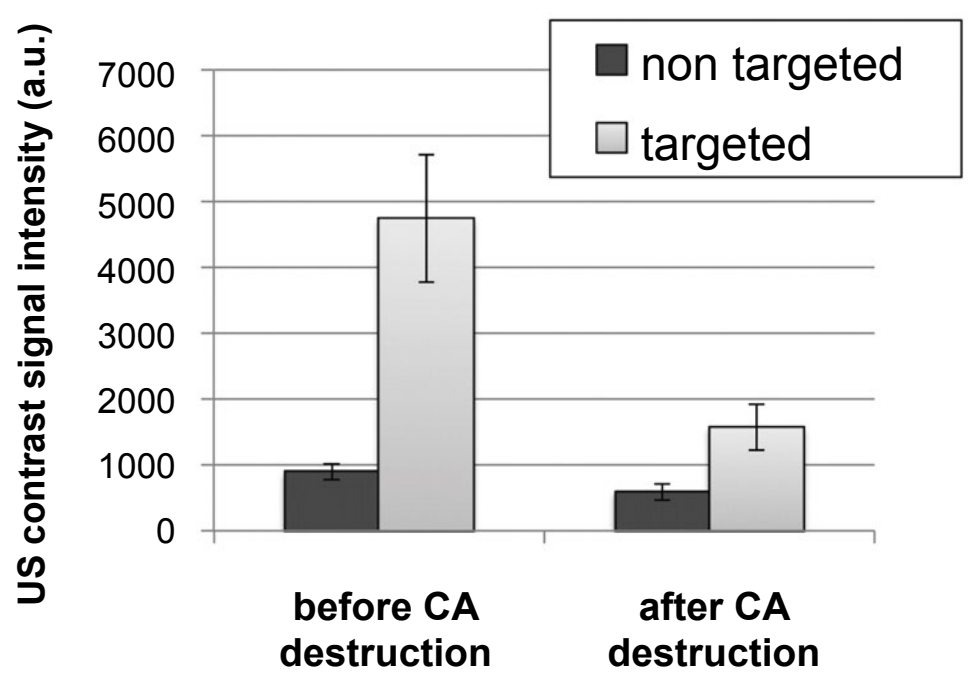

Figure 5: Mean $( \pm$ SE) US contrast signal intensities evaluated 10 minutes after the CA injection, before and after targeted and non-targeted CA destruction. Non-significant difference was observed in the signal for non-targeted CA, whereas a significant decrease was observed for the targeted CA ( $p=0.03$, Wilcox on test), indicating the binding of the specific CA. DTE values (mean \pm SE), calculated as the difference between US signal intensities before and after CA destruction, were $260.5 \pm 66.0$ a.u. for the non-targeted CA and $2601.5 \pm 488.3$ a.u. for the targeted CA. Significantly different changes in contrast were observed between US targeted and non-targeted CA ( $p=0.002$, Wilcox on test for paired samples).

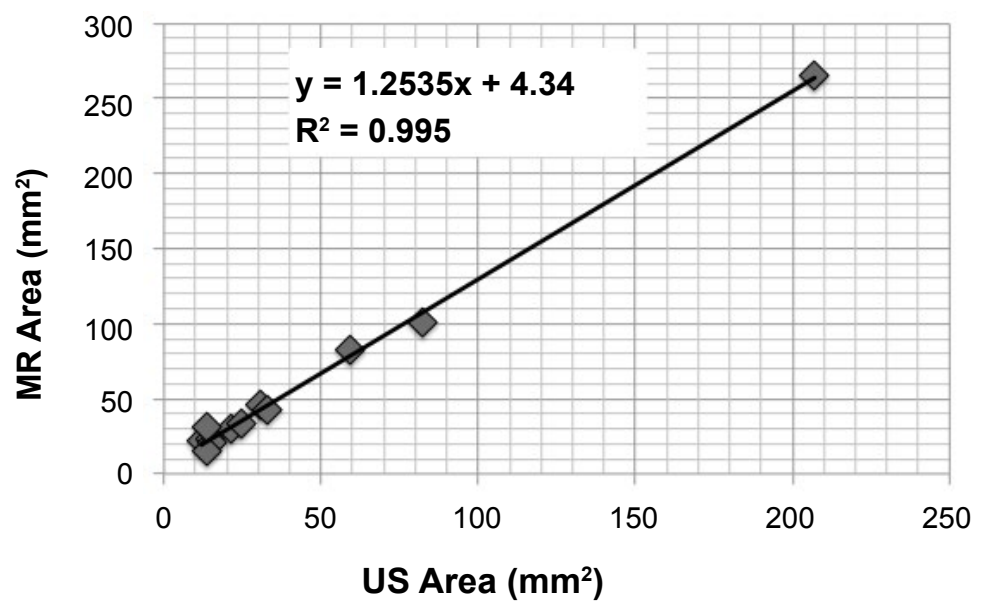

Figure 6: Tumoral size evaluation from MR and US imaging, where $r=0.99, p<0.001$ (Pearson test). The tumor area measured from MR imaging was $25 \%$ higher than that estimated using US. 


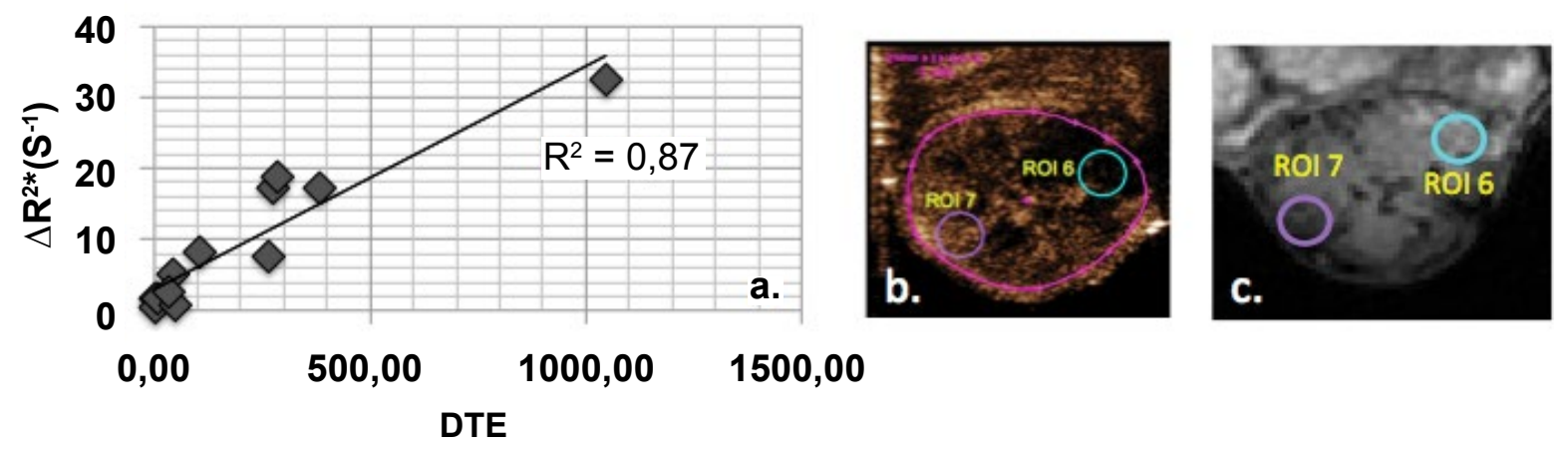

Figure 7: a) Maximum correspondence between US and MR parameters $\left(r=0.875\right.$, Pearson test $\left.p<1.10^{-5}\right)$ was observed for the specific ROIs, i.e., the hypo- and hyper-vascularized areas (ROI 6 and ROI 7), defined in b) US and c) MR acquisitions at 60 minutes after injection.

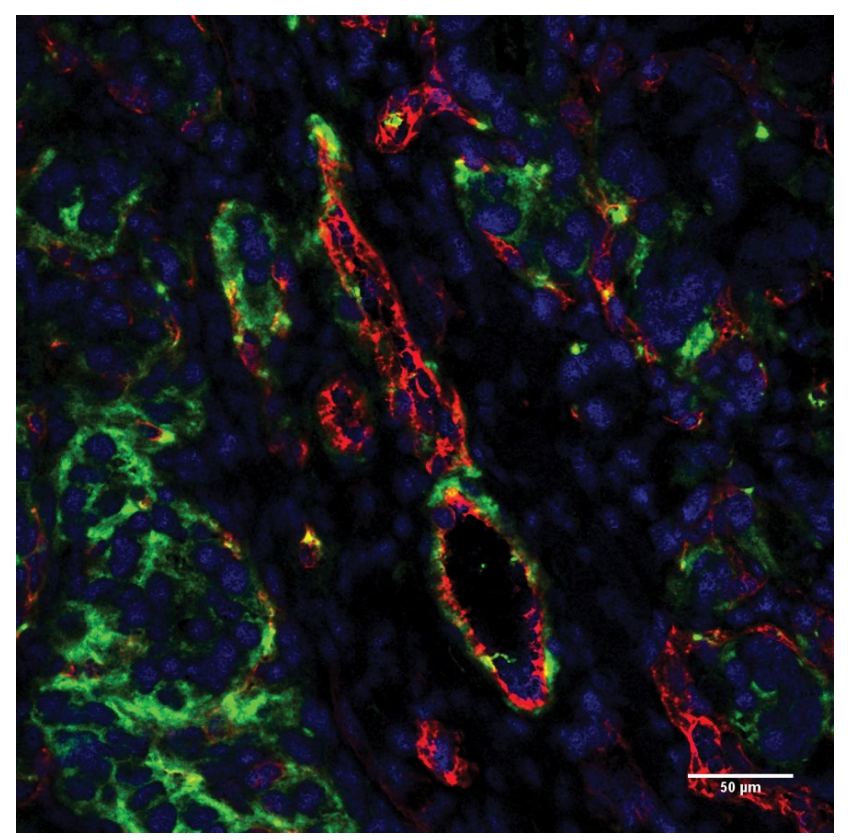

Figure 8: Examples of immunofluorescence merged images for $\beta 3$ (green), CD31 (red), and DAPI (blue) obtained using multi photon microscopy in A498 tumor sections. Bar $=50 \mu \mathrm{m}$.

tion. The signal did not change in the case of non-targeted CA, but a significant decrease was observed for the targeted CA ( $p=0.03)$, indicating the binding of the

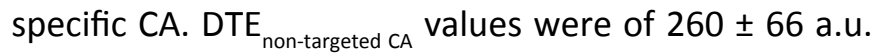
compared to $2601 \pm 488$ a.u for $D_{T E} E_{\text {targeted } C A^{\prime}}$ indicating significantly different contrast enhancement $(p=0.002)$.

\section{Molecular US and MR colocalization}

The manually segmented tumor areas correlated well between the two imaging modalities $(r=0.99$, $p<0.001$, Figure 6), as expected. The tumor area measured using MR-imaging was $25 \%$ larger than the tumor area measured using US; this difference may be due to different manual tracing.

The correlation coefficient $r$ was evaluated for both $\Delta R_{2}{ }^{*}$ and DTE values measured in all seven specific ROIs for the different post-injection times in the DSC-MR protocol. The best correlations in all the ROls were obtained at 60 minutes postinjection, with $r$ values of 0.52
( $p=0.0005)$. The mean values of $\Delta R_{2}{ }^{*}$ and DTE measured for ROI 1 to 5 were not significantly correlated, even for different post-injection times $(r<0.71)$. The strong correlation was observed on the specific ROIs, i.e., the hypo- and hyper-vascularized areas (ROI 6 and $\mathrm{ROI} 7$, Figure 7 with respectively $r_{10 \min }=0.86(p=0.0003) ; r_{30 \mathrm{~min}}$ $=0.85(p=0.0004)$ and $r_{60 \min }=0.87(p=0.0001)$.

\section{Immunofluorescence}

The observations acquired by optical microscopy in tissue images (Figure 8 ) indicated the colocalization of $\beta 3$ with $C D 31$, confirming the presence of integrin on the endothelial cells of this A498 RCC tumor model. Several integrins are involved in cancer progression but most of them, such as $\alpha v \beta 3$, are over expressed, making them difficult to quantify $[6,11,12]$. We successfully evaluated colocalization using Imaris (Bit plane Scientific Software) to quantify the percentage of colocalization between the 2 CD31 and $\beta 3$ markers, and found that colocalized $\beta 3 / C D 31$ areas made up $30.6 \%$ of the vasculature area.

\section{Discussion}

The contrast agents, i.e. iron oxide nanoemulsion (DSC-MR) and microbubbles (DTE-US), were both functionalized to target the same integrin $(\alpha \vee \beta 3)$ for preclinical oncology applications, and both were shown to provide specific targeting [13]. The novelty of this work was to make a fair comparison of molecular imaging approaches seeking to identify the colocalization of angiogenesis target, using different contrast agents with different fixation mechanisms. These results highlight the reliability of molecular imaging for angiogenesis.

An entire analysis protocol was specifically developed here to colocalize the US 2D acquisition to the closest slice in the 3D MR-acquisition volume. This protocol was combined with a systematic segmentation of the different tumor areas where $\Delta R_{2}{ }^{*}$ and DTE were calculated. In this way, we ensured the closest and thus fairest possible comparison between MR and US results, enabling region-based comparison among imaging modalities.

First, the comparison of these specific contrast agents 
with the non-targeted contrast agents within each imaging session yielded significant differences (MR - Figure 4, US - Figure 5). The dynamic contrast provided by MR imaging allowed the evaluation to take place over a sufficiently long time scale to observe the CA kinetic, which was not the case for US acquisitions. US experiments gave access to measurement of the quantity of targeted microbubbles at only one time point, i.e. 10 minutes postinjection, as recommended by Van Rookie, et al. [14]. The optimal window to perform MR imaging was assessed (Figure 4), and the targeted MR signal became significantly different from the non-targeted one after 37 minutes. The MR CA injected for this study had larger diameters and stronger relaxivity than those commonly used, and appears to be a promising tool for molecular imaging. These molecular MR CAs, based on nano-objects and containing a high payload of contrast-generating metals (USPIO in this study), are now sensitive enough for use in target-specific imaging. In light of the sensitivity $\left(r_{2}^{*} \sim 210 \mathrm{mM}^{-1} \cdot \mathrm{s}^{-1}\right)$ and the observed average specific $\Delta \mathrm{R}_{2}^{*}$ (close to $10 \mathrm{~s}^{-1}$ ) recorded, we can consider that approximately $50 \mu \mathrm{M}$ concentration of iron (or a 50 pM concentration of droplets) was measurable, which is within the range of receptor concentrations.

Secondly, a good correlation was observed between specific targeted MR and US signal, with a high correlation coefficient of 0.87 at 60 minutes after injection Figure 7.

The results highlighted the high colocalization of $\mathrm{MR}$ and US targeted contrast agents in vivo, despite differences in their respective sizes (190 nm vs. 2-3 $\mu \mathrm{m}$ diameters) and fixation mechanisms. MR nanoemulsion was targeted with RGD, while US CA was targeted with antibodies. Thus, the high colocalization observed in this study demonstrated the reliability of molecular imaging, whatever imaging modalities, MR or US. However, simultaneous imaging by both modalities does not provide any evidence that could explain the fundamental biological fixation mechanism. In particular, the different CA sizes led us to expect the observation of some integrins expressed exclusively on activated endothelial cells from vessels with the DTE-US, and integrins expressed on tumor cells after extravasation of the MR $A C$. These differences in CA behavior might explain observations made in other ROIs.

The localization of $\beta 3$ was assessed and confirmed by immunofluorescence within the vessels and within the extracellular matrix. The difficulty of implementing integrin labelling and observation makes it impossible to study slices thicker than 20 to $30 \mu \mathrm{m}$, which would allow a more robust observation and possible quantification within the vessels. Nevertheless, the multi-staining DAPI/CD31/ß33 was optimized to allow high quality visualization.

Finally, our study highlights the equivalence of molecular DTE-US and DSC-MR imaging in terms of specificity and CA localization. This approach has already allowed the prediction of response to targeted therapies by the use of MR and US imaging to evaluate the early response of different therapeutic agents in renal cell carcinoma [15]. Some studies suggested that RGD peptide could not necessarily reflect the change of integrin $\alpha v \beta 3$ expression on neoangiogenesis detection $[16,17]$. Consequently, the real potential of RGD peptide in therapy response monitoring needs to be confirmed with well-designed clinical investigations Beyond this limit of targeted-CAs specificity, the main benefits of the molecular US approach are its accessibility and low cost. Limitations linked to operator dependency can often be overcome with practice. The evaluation of early response to treatment could be achieved by improving methods for the detection and evaluation of tumors and identifying the expression levels of cell surface receptor such as integrins, thus permitting doctors to select the most efficient type of treatment for each case. Combining this early response to treatment with an accessible imaging technique such as molecular US would be a major gain for personalized care.

\section{Acknowledgments}

This work was performed on platforms affiliated to the France Life Imaging network (grant ANR-11-INBS-0006), and was partially funded by the network.

\section{References}

1. Kiessling F, Contag $\mathrm{CH}$ (2016) World Molecular Imaging Congress 2016: Imaging Biology-Improving Therapy. Mol Imaging Biol 18: 313-314.

2. Deshpande N, Needles A, Willmann JK (2010) Molecular ultrasound imaging: current status and future directions. Clin Radiol 65: 567-581.

3. Kiessling F, Fokong S, Koczera P, Lederle W, Lammers T (2012) Ultrasound microbubbles for molecular diagnosis, therapy, and theranostics. J Nucl Med 53: 345-348.

4. Massoud TF, Gambhir SS (2003) Molecular imaging in living subjects: Seeing fundamental biological processes in a new light. Genes Dev 17: 545-580.

5. Weissleder R, Mahmood U (2001) Molecular imaging. Radiology 219: 316-333.

6. Avraamides CJ, Garmy-Susini B, Varner JA (2008) Integrins in angiogenesis and lymphangiogenesis. Nat Rev Cancer 8: 604-617.

7. Poirier-Quinot $M$, de Rochefort $L$, Girard $O$, Darrasse $L$ (2013) MRI: recent advances and new horizons.

8. Weissleder R, Pittet MJ (2008) Imaging in the era of molecular oncology. Nature 452: 580-589.

9. Leguerney I, Scoazec JY, Gadot N, Robin N, Pénault-Llorca F, et al. (2015) Molecular ultrasound imaging using contrast agents targeting endoglin, vascular endothelial growth factor receptor 2 and integrin. Ultrasound Med Biol 41: 197-207.

10. Willmann JK, Paulmurugan R, Chen K, Olivier Gheysens, Martin Rodriguez-Porcel, et al. (2008) US imaging of tumor angiogenesis with microbubbles targeted to vascular endothelial growth factor receptor type 2 in mice. Radiology 246 : 508-518.

11. Desgrosellier JS, Cheresh DA (2010) Integrins in cancer: biological implications and therapeutic opportunities. Nat Rev Cancer 10: 9-22. 
12. Yan F, Xu X, Chen Y, Deng Z, Liu H, et al. (2015) A Lipopeptide-Based $\alpha v \beta_{3}$ Integrin-Targeted Ultrasound Contrast Agent for Molecular Imaging of Tumor Angiogenesis. Ultrasound Med Biol 41: 2765-2773.

13. Niu G, Chen $X$ (2011) Why integrin as a primary target for imaging and therapy. Theranostics 1: 30-47.

14. van Rooij T, Daeichin V, Skachkov I, de Jong N, Kooiman K (2015) Targeted ultrasound contrast agents for ultrasound molecular imaging and therapy. Int J Hyperthermia 31: 90-106.
15. Leguerney I, de Rochefort L, Poirier-Quinot M, Alexandre Ingels, Xavier Violas, et al. (2017) Molecular imaging to predict response to targeted therapies in renal cell carcinoma. Contrast Media \& Molecular Imaging 2017: 7498538.

16. Rylova SN, Barnucz E, Fani M, Friederike Braun, Martin Werner, et al. (2014) Does imaging avb3 integrin expression with PET detect changes in angiogenesis during bevacizumab therapy? J Nucl Med 55: 1878-1884.

17. Niu G, Chen XY (2016) RGD PET: From lesion detection to therapy response monitoring. J Nucl Med 57: 501-502. 